\title{
Localization-Delocalization Transition for Drift Diffusion in a Random Environment
}

\author{
Paul C. Bressloff,* Vincent M. Dwyer, ${ }^{\dagger}$ and Michael J. Kearney ${ }^{\dagger}$ \\ Loughborough University, Loughborough, Leics. LE11 3TU, United Kingdom
}

(Received 23 August 1996)

\begin{abstract}
We investigate the localization-delocalization transition for the drift-diffusion equation on a regular tree with quenched random drift velocities on its branches. The inverse of the steady-state amplitude at the origin is expressed in terms of a random geometric series whose convergence or otherwise determines the critical phase boundary. We establish exact criteria for localization valid for an arbitrary distribution of the drift velocities. The phase transition is shown to be first order except in the percolation limit. [S0031-9007(96)01870-4]
\end{abstract}

PACS numbers: 64.60.Cn, 05.40.+j, 05.60.+w

There are a number of interesting physical problems involving transport in quenched random environments. These include sedimentation in porous media, diffusion limited aggregation, directed polymers, and electromigration along grain boundary networks (see, e.g., [1,2], and references therein). Random walks in random environments have attracted a great deal of attention as models of such systems. Of particular interest has been the study of how the competition between drift and diffusion in asymmetric random walks can lead to anomalous diffusive behavior [3-11]. However, there are comparatively few exact results known concerning the corresponding transport properties in continuum random media. In this Letter we investigate the localization-delocalization transition (in a sense to be made clear below) of the drift-diffusion equation on a regular tree with quenched random drift velocities on its branches. Given an arbitrary distribution of the drift velocities, we establish exact criteria to determine whether the system is localized or delocalized.

Consider a regular tree $\Gamma$ with branching number $z$ and segment length $L=1$ (Fig. 1). It is convenient to partition the branches of the tree into successive generations. The first generation $\Sigma_{1}$ consists of the $z$ branches connected to the origin, the second generation $\Sigma_{2}$ consists of the $z^{2}$ subsequent branches connected to the first generation, and so on. The $n$th generation contains $z^{n}$ branches. The set of branches in one generation connected to a segment $i$ in the preceding generation is denoted by $I_{i}$. The concentration $c_{i}(x, t)$ at position $x$ and time $t$ on the $i$ th segment of the tree evolves according to the equation

$$
\frac{\partial c_{i}}{\partial t}=\frac{\partial^{2} c_{i}}{\partial x^{2}}+v_{i} \frac{\partial c_{i}}{\partial x}, \quad t>0, \quad 0<x<1,
$$

with the end closer to the origin of the tree chosen to be at $x=0$. Here the diffusion constant $D$ is taken to be the same on every branch $(D=1)$ and $v_{i}$ is the drift velocity, which is taken to be positive if directed towards the origin, that is, in the negative $x$ direction. Equation (1) is supplemented by the boundary conditions expressing continuity of the concentration at a node

$$
\begin{array}{ll}
c_{i}(0, t)=c_{j}(0, t), & i, j \in \Sigma_{1}, \\
c_{i}(1, t)=c_{j}(0, t), & j \in I_{i}
\end{array}
$$

and conservation of current through the node

$$
\sum_{k \in \Sigma_{1}} J_{k}(0, t)=0, \quad J_{i}(1, t)=\sum_{k \in I_{i}} J_{k}(0, t),
$$

where $J_{i}(x, t)=-\partial c_{i} / \partial x-v_{i} c_{i}(x, t)$.

In steady state the current vanishes on each segment $J_{i} \equiv 0$ so that the solution is of the form $c_{i}(x)=A_{i} e^{-v_{i} x}$. The continuity conditions (2) imply that the amplitudes $A_{i}$ satisfy the iterative equation $A_{i}=\Phi$ for $i \in \Sigma_{1}$ and $A_{i} e^{-v_{i}}$ for all $i$ and $j \in I_{i}$ where $\Phi$ is the steady-state concentration at the origin. Thus the amplitude $A_{i}$ on a given segment $i \in \Sigma_{n}, n>1$ may be expressed in terms of the concentration at the origin according to the relation $A_{i}=\left[\prod_{j<i} e^{-v_{j}}\right] \Phi$ where $\{j, j<i\}$ denotes the unique sequence of segments joining $i$ to the origin. Assuming that the initial concentration is normalized to unity, conservation of particle number implies that $\sum_{i} \int_{0}^{1} c_{i}(x) d x=$ 1. Making the substitution $c_{i}(x)=A_{i} e^{-v_{i} x}$ the yields the following equation for $\Phi$ :

$$
\begin{aligned}
\Phi^{-1}=\sum_{i \in \Sigma_{1}}( & f\left(v_{i}\right)+g\left(v_{i}\right) \sum_{j \in I_{i}} f\left(v_{j}\right) \\
& \left.+g\left(v_{i}\right) \sum_{j \in I_{i}} g\left(v_{j}\right) \sum_{k \in I_{i}} f\left(v_{k}\right)+\cdots\right),
\end{aligned}
$$

where $f(v)=\left(1-e^{-v}\right) / v, g(v)=e^{-v}$. We shall assume that $v$ is finite so that $f(v), g(v)$ are bounded, positive functions.

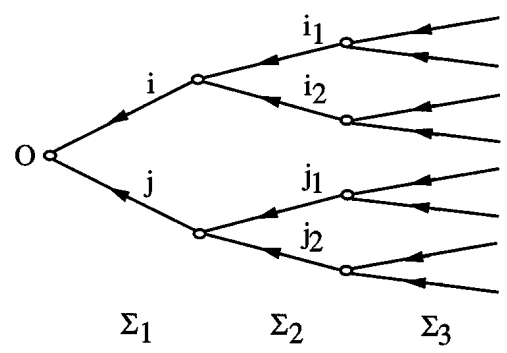

FIG. 1. Topologically biased regular tree with branching number $z=2$ indicating successive generations $\Sigma_{1}=\{i, j\}$, $\Sigma_{2}=\left\{i_{1}, i_{2}, j_{1}, j_{2}\right\}$, etc. Also $I_{i}=\left\{i_{1}, i_{2}\right\}$, etc. Arrows indicate direction of the drift velocity $v_{i}$ on each branch $i$ relative to the origin $O$. 
Equation (4) expresses $\Phi^{-1}$ in terms of an infinite series. If this series is convergent then $\Phi$ has a finite value and the steady state is localized. On the other hand, if the series diverges then $\Phi=0$ and the steady state is delocalized. The simplest case to analyze is when all drift velocities are the same, $v_{i}=v$ for all $i$, so that we have a homogeneous tree. Then Eq. (4) reduces to the geometric series

$$
\Phi^{-1}=f(v) \sum_{p=0}^{\infty} z^{p+1} g(v)^{p}=\frac{z f(v)}{1-z g(v)},
$$

provided that $z g(v)<1$. This yields a critical drift velocity $v_{c}=\ln z$ beyond which the system is localized. If $v<$ $v_{c}$ then the asymptotic decay of the delocalized state exhibits conventional behavior, whereas at the critical point $v=v_{c}$ there is anomalous behavior in the form of a critical slowing down [12]. In this Letter we are interested in deriving conditions for localization when the drift velocities $v_{i}$ are quenched random variables. Time-dependent aspects of the problem will not be addressed here.

Let us begin by examining a simplified but still interesting version of the full problem. Suppose that within each generation $n$ all segments have the same drift velocity $v_{n}$ but the sequence $\left\{v_{n}, n \geq 1\right\}$ is independently and identically distributed (intergenerational disorder). Let $\rho(v)$ be the associated probability density. Equation (4) simplifies to the form

$$
(z \Phi)^{-1} \equiv R=\sum_{n=1}^{\infty} f\left(v_{n}\right) z^{n-1} \prod_{m=1}^{n-1} g\left(v_{m}\right)
$$

so that the steady-state concentration is expressed in terms of a random geometric series $R$. Similar series have arisen in a variety of studies of one-dimensional problems $[1,3-$ $8,13]$. It can be shown that if $\langle\ln [z g(v)]\rangle<0$ then $R$ converges with probability one [7]. Hence the steady state is localized provided that $\langle\boldsymbol{v}\rangle>\boldsymbol{v}_{c}=\ln z$, that is, the average drift velocity exceeds the critical velocity for localization on a homogeneous tree. On the other hand, if $\langle\boldsymbol{v}\rangle\left\langle\boldsymbol{v}_{c}\right.$ then $R$ is infinite and the steady state is delocalized. Using Jensen's inequality $\left\langle e^{v}\right\rangle \geq e^{\langle v\rangle}$, it is simple to show that this localization criterion is stronger than that based on the vanishing of the asymptotic particle velocity, which takes the form $\langle 1 / g(v)\rangle>z$. The latter is a necessary but not sufficient condition for localization [12].

In the language of phase transitions, there is a transition from a localized to a delocalized state at the critical points

$$
\langle v\rangle>\ln z \quad \text { (localized), } \quad\langle v\rangle<\ln z \quad \text { (delocalized). }
$$

The critical points determine a phase boundary in the infinite-dimensional space of probability densities $\rho(v)$ that separates the localized and delocalized phases. A characteristic feature of the phase transition is that as $\langle v\rangle \rightarrow v_{c}$ in some prescribed fashion the probability distribution $F$ of $R$ in the localized phase develops a long tail for which all moments are infinite. This is a conse- quence of the fact that when the first moment $\int r d F(r)=$ $\langle f(v)\rangle /[1-z\langle g(v)\rangle]$ becomes infinite, $\langle g(v)\rangle=1 / z$, the system is still localized since, from Jensen's inequality, $\langle v\rangle \geq v_{c}$. Assuming the existence of a probability density $\Psi(r)$ such that $d F(r)=\Psi(r) d r$, we obtain the following integral equation for $\Psi$ :

$$
\Psi(r)=\int_{-\infty}^{\infty} \frac{\rho(v)}{z g(v)} \Psi\left(\frac{r-f(v)}{z g(v)}\right) d v .
$$

This equation can be derived from the renewal equation $Y_{n}=z g\left(v_{n}\right) Y_{n+1}+f\left(v_{n}\right)$ with each pair $\left(f\left(v_{n}\right), g\left(v_{n}\right)\right)$ generated independently from $\rho(v)$ and $Y_{0}$ fixed; the distribution of $Y_{n}$ converges to that of $R$ independently of $Y_{0}$ [7]. An alternative form of the integral equation (8) is obtained by taking Laplace transforms:

$$
M(s)=\int_{-\infty}^{\infty} \rho(v) M(z g(v) s) e^{-s f(v)} d v,
$$

with $M(s)=\int_{0}^{\infty} e^{-s r} \Psi(r) d r$. It is not generally possible to solve these equations analytically. However, one can determine the asymptotic behavior of $\Psi$ when $r$ is large. Suppose that $\rho(v)$ is nonarithmetic, that is, $\rho(v)$ cannot be written in the form $\sum_{n=-\infty}^{\infty} p_{n} \delta\left(v-\lambda^{n}\right)$ for any $\lambda$ and $\left\{p_{n}\right\}$ such that $\sum_{n=-\infty}^{\infty} p_{n}=1$. Also assume that the first moment of $\Psi$ is infinite so that $\langle g(v)\rangle>1 / z$. It can then be proven $[7,8,13]$ that there exist positive constants $a, \sigma$ with $0<\sigma<1$ such that $\Psi(r) \sim a r^{-\sigma-1}$ for large $r$ [and so $M(s) \sim 1+b s^{\sigma}$ for small $s$ ]. In other words, $Y_{n}$ is in the domain of attraction of a Levy stable law. The large- $r$ behavior of $\Psi$ ensures that if $\sigma>0$ then $F^{*} \equiv$ $\lim _{y \rightarrow \infty} \int_{y}^{\infty} d F(r)=0$, in other words, that the series $R$ of Eq. (6) is convergent with probability one. Substitution of the asymptotic form for $\Psi$ (or $M$ ) into Eq. (8) [or (9)] leads to the equation $G(\sigma) \equiv z^{\sigma}\left\langle g(v)^{\sigma}\right\rangle-1=0$. Provided that the density $\rho(v)$ satisfies the previously stated conditions, there exist two real roots of $G(\sigma)$, $\sigma=0, \bar{\sigma}$, and the real part of all complex roots is larger than $\bar{\sigma}$. Hence the nontrivial real root $\bar{\sigma}$ dominates for large $r$. Suppose that $\rho(v)$ depends smoothly on some parameter $\lambda$ such that $\bar{\sigma}(\lambda)>0$ for $\lambda<\lambda_{c}$ and $\lim _{\lambda \rightarrow \lambda_{c}} \bar{\sigma}(\lambda)=0$. In the limit $\lambda \rightarrow \lambda_{c}, \Psi$ ceases to exist (it is no longer normalizable) and the probability $F^{*}$ that $R$ is infinite jumps from $F^{*}=0$ to $F^{*}=1$. Identifying $F^{*}$ as an order parameter we deduce that the localization-delocalization phase transition is first order. Differentiating the equation $G(\bar{\sigma}(\lambda), \lambda)=0$ with respect to $\lambda$ gives

$$
\begin{gathered}
\int \frac{d \rho_{\lambda}(v)}{d \lambda}[z g(v)]^{\bar{\sigma}(\lambda)} d v+ \\
\bar{\sigma}^{\prime}(\lambda)\left\langle[z g(v)]^{\bar{\sigma}(\lambda)} \ln [z g(v)]\right\rangle_{\lambda}=0 .
\end{gathered}
$$

Taking the limit $\lambda \rightarrow \lambda_{c}$ in Eq. (10) leads to the result $\bar{\sigma}^{\prime}\left(\lambda_{c}\right)\left[\langle v\rangle_{\lambda_{c}}-v_{c}\right]=0$. Since $\lambda_{c}$ is a bifurcation point it follows that $\bar{\sigma}^{\prime}\left(\lambda_{c}\right)>0$ and hence $\langle v\rangle_{\lambda_{c}}=v_{c}$. 
The restriction to intergenerational disorder reduced the analysis of the steady state to an effective onedimensional problem. This is no longer the case when one has intragenerational disorder where the topology of the tree must be taken into account explicitly. Suppose that the drift velocity on each segment is now independently and identically distributed with a probability density $\rho(v)$. The resulting random series on the right-hand side of Eq. (4) is then generated by a renewal process on the tree. Consider a bounded tree of $N$ generations and associate with each segment $i$ a random variable $Y_{i}^{(N)}$ such that (for fixed $Y_{k}^{(N)}, k \in \Sigma_{N}$ )

$$
\begin{gathered}
Y_{i}^{(N)}=\sum_{j \in I_{i}} g\left(v_{i}\right) Y_{j}^{(N)}+f\left(v_{i}\right), \\
i \in \Sigma_{n}, 1 \leq n<N .
\end{gathered}
$$

Equation (4) may then be rewritten in the form

$$
\Phi^{-1}=\sum_{i \in \Sigma_{1}} R_{i}, \quad R_{i}=\lim _{N \rightarrow \infty} Y_{i}^{(N)} .
$$

Suppose that $R_{i}$ converges with probability one independently of the boundary conditions. The symmetry of the tree then ensures that all variables $R_{i}, i \in \Sigma_{1}$, are identically and independently distributed with a probability distribution $F$. The renewal equation (11) implies that the associated probability density $\Psi$ satisfies the fixed point equation

$$
\begin{aligned}
\Psi(y)= & \int_{0}^{\infty} \prod_{j=1}^{z} \Psi\left(y_{j}\right) d y_{j} \int_{-\infty}^{\infty} \rho(v) \\
& \times \delta\left(y-g(v) \sum_{j=1}^{z} y_{j}-f(v)\right) d v
\end{aligned}
$$

Laplace transforming Eq. (13) gives a corresponding integral equation for the generating function $M(s)$ :

$$
M(s)=\int_{-\infty}^{\infty} \rho(v)[M(s g(v))]^{z} e^{-s f(v)} d v .
$$

Note that when $z=1$ Eq. (14) reduces to Eq. (9); there is no distinction between intergenerational and intragenerational disorder in one dimension.

Suppose that we expand the generating function $M(s)$ for small $s$ along similar lines to the intergenerational case such that $M(s) \sim 1+b s^{\sigma}$. Substituting into Eq. (14) yields the equation $H(\sigma) \equiv z\left\langle g(v)^{\sigma}\right\rangle-1=0$. In contrast to the function $G(\sigma), \sigma=0$ is not an allowed root of $H(\sigma)=0$. Therefore the transition is no longer characterized by the limit $\sigma \rightarrow 0$. Introduce the index $\sigma^{*} \in[0,1]$ defined according to the property $H\left(\sigma^{*}\right)=$ $\min _{0 \leq \sigma \leq 1} H(\sigma)$. Note that $\sigma^{*}$ depends on the branching number $z$ and the probability density $\rho(v)$. If $H\left(\sigma^{*}\right)>0$ then any solution of $H(\sigma)=0$ must satisfy $\sigma>1 \mathrm{im}-$ plying that the first moment of $\Psi$ is finite. On the other hand, $H(1)>0$ implies that the first moment is infinite. The evident contradiction shows that if $H\left(\sigma^{*}\right)>0$ then the only allowed solution of the integral equation (14) for $s>0$ is $M(s)=0$ and the system is delocalized. We infer from this and our analysis of intergenerational disorder that there is a first-order phase transition from a localized to a delocalized state at the critical points $\sigma_{c}^{*}>0$ where $H\left(\sigma_{c}^{*}\right)=0$. This determines a phase boundary in the space of probability densities that separates the localized and delocalized phases:

$$
\begin{array}{ll}
z\left\langle e^{-\sigma^{*} v}\right\rangle<1 & \text { (localized), } \\
z\left\langle e^{-\sigma^{*} v}\right\rangle>1 & \text { (delocalized). }
\end{array}
$$

Strictly speaking, the above analysis of the integral equation (14) has established only the second inequality in Eq. (15). In the case of distributions that allow only positive drift velocities [so that $0<g(v)<1$ ] one can also establish the first inequality from Eq. (14) since $H(\sigma)$ is then a monotonically decreasing function of $\sigma$. The latter implies that if $H\left(\sigma^{*}\right)<0$ then $H(1)<0$, and hence that all moments of $\Psi$ are finite, i.e., the system is localized. It also follows that the system becomes delocalized as soon as the first moment of $\Psi$ becomes infinite and hence $\Psi$ does not develop a long tail near the transition point. A more rigorous derivation of the two inequalities in Eq. (15), which proves they hold for arbitrary distributions of the drift velocities, will be presented elsewhere using some recent powerful theorems concerning random walks on trees $[14,15]$.

To illustrate the above ideas we shall consider a particular example of the velocity probability density $\rho(v)$, namely, a Gaussian with mean $\mu$ and variance $\Delta^{2}$. In Fig. 2 the resulting phase boundary for intragenerational disorder is displayed in the parameter space given by the mean and variance (the curve separating regions II and III). This boundary is determined by finding $\sigma^{*}$ for a given pair $(\mu, \Delta)$ and then solving the equation $z\left\langle e^{-\sigma^{\prime \prime} v}\right\rangle=1$ to obtain $\Delta$ as a function of $\mu$. One finds that $\sigma^{*}=\mu / \Delta^{2}$ if $\mu<\Delta^{2}$ and $\sigma^{*}=1$ if $\mu \geq \Delta^{2}$. This leads to the following explicit expression for the phase boundary: $\Delta(\mu)=\sqrt{2(\mu-\ln z)}$ for $\mu \in[\ln z, 2 \ln z]$

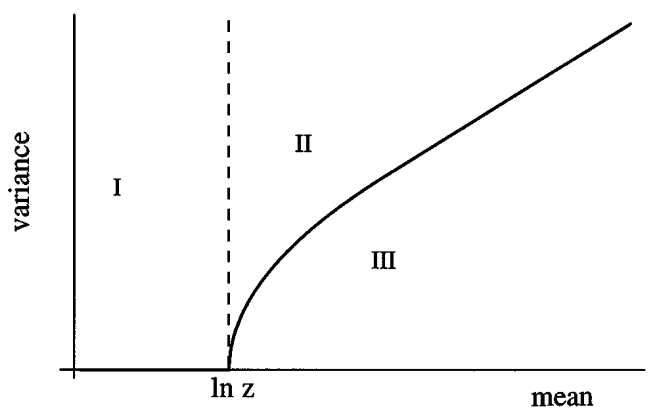

FIG. 2. Phase diagram showing localization-delocalization phase boundaries for a Gaussian distribution of the drift velocities. In the case of intergenerational disorder the system is delocalized in region I and localized in regions II and III, whereas in the case of intragenerational disorder the system is localized only in region III. 
and $\Delta(\mu)=\mu / \sqrt{2 \ln z}$ for $\mu>2 \ln z$. The corresponding phase boundary for intergenerational disorder is the vertical line through $\mu=\ln z$ and is independent of the variance. The physical interpretation of these results is that the removal of correlations within a generation when going from the intergenerational to the intragenerational case leads to a higher tendency to delocalize.

So far we have assumed that the drift velocities are finite so that $0<g(v)<\infty$. If there exists a nonzero probability that $v$ is infinite $[g(v)=0]$ then (in the case of intragenerational disorder) we have a generalized bond percolation problem [2,14] since any branch $i$ that has an infinite drift velocity acts as a broken bond. In such a circumstance one can show that the transition is second order rather than first order. The system is still localized when $z\left\langle e^{-\sigma^{*} v}\right\rangle<1$ with $F^{*}=0$. However, if $z\left\langle e^{-\sigma^{*} v}\right\rangle>1$ then $0<F^{*}<1$ so that there is a nonzero probability that the random series $R_{i}$ of Eq. (12) is finite and hence a nonzero probability that the system is localized beyond the critical point. To illustrate this we shall consider conventional bond percolation for which $\rho(v)$ is taken to be a Bernoulli distribution $\rho(v)=p \delta(v)+(1-p) \delta(v-\bar{v})$ with $\bar{v} \rightarrow \infty$. It follows immediately from Eq. (15) that the percolation threshold is $p_{c}=1 / z$. The integral equation (14) becomes $M(s)=p M(s)^{z} e^{-s}+(1-p)$ since $M(0)=1$. In the particular case $z=2$ one obtains

$$
M(s)=e^{s}\left[1-\sqrt{1-4(1-p) p e^{-s}}\right] / 2 p,
$$

from which we deduce that $1-F^{*} \equiv \lim _{s \rightarrow 0} M(s)=$ $(1-|2 p-1|) / 2 p$. For $p<1 / 2$ we have $F^{*}=0$, whereas for $p>1 / 2$ we have $F^{*}=(2 p-1) / p$. Of course, such results are well known [2] although it is interesting to see them derived in a different context.

In summary, we have derived exact criteria to determine the localization-delocalization phase boundary for the steady-state solution of the drift-diffusion equation on a regular tree with quenched random drift velocities on its branches. Three cases have been considered: (i) uniform drift for which the criterion is $v=\ln z$, (ii) intergenera- tional disorder for which the criterion is $\langle v\rangle=\ln z$, and (iii) full intragenerational disorder for which the criterion is $z\left\langle e^{-\sigma^{*} v}\right\rangle=1$ with a suitably defined index $\sigma^{*}$. Certain results in this Letter can be modified to handle trees that are not homogeneous in branching number [14]. Another interesting issue concerns the localization length $\xi$ which, from Eq. (4), we assume satisfies the scaling law (for large $n$ ) $\Phi \exp \left[-(n / \xi)^{\nu}\right]=\left\langle\sum_{j \in \Sigma_{n}} A_{j}\right\rangle$ for some exponent $\nu$. In the case of intergenerational disorder $\xi$ is self-averaging and $\xi^{-1}=\langle v\rangle-\ln z$ with $\nu=1$. The corresponding result for intragenerational disorder is more difficult to establish and the localization length may no longer be a self-averaging quantity. These and other issues will be discussed in more detail elsewhere.

*Department of Mathematical Sciences.

${ }^{\dagger}$ Department of Electronic and Electrical Engineering.

[1] J.-P. Bouchaud and A. Georges, Phys. Rep. 195, 127 (1990).

[2] Fractals and Disordered Systems, edited by A. Bunde and S. Havlin (Springer-Verlag, Heidelberg, 1991).

[3] F. Solomon, Ann. Probab. 3, 1 (1975).

[4] H. Kesten, M. V. Kozlov, and F. Spitzer, Compos. Math. 30, 169 (1975).

[5] Ya G. Sinai, Lect. Notes Phys. 153, 12 (1982).

[6] B. Derrida and Y. Pomeau, Phys. Rev. Lett. 48, 627 (1982).

[7] H. Kesten, Acta Math. 131, 208 (1973).

[8] C. de Calan, J.M. Luck, Th. M. Nieuwenhuizen, and D. Petritis, J. Phys. A 18, 501 (1985).

[9] D. Cassi, Europhys. Lett. 9, 637 (1989).

[10] C. Aslangul, M. Barthélémy, N. Pottier, and D. SaintJames, Europhys. Lett. 15, 251 (1991).

[11] A. Bunde, S. Havlin, and H. E. Roman, Phys. Rev. A 42, 6274 (1990).

[12] P.C. Bressloff, V.M. Dwyer, and M. J. Kearney, J. Phys. A 29, 6161 (1996).

[13] B. Derrida and H. J. Hilhorst, J. Phys. A 16, 2641 (1983).

[14] R. Lyons, Ann. Prob. 18, 931 (1990).

[15] R. Lyons and R. Pemantle, Ann. Prob. 20, 125 (1992). 